\title{
Superficial Siderosis of the Central Nervous System Originating from the Thoracic Spine: A Case Report
}

\author{
Sung Mo Ryu, Eun-Sang Kim, Seung-Kook Kim, Sun-Ho Lee, Whan Eoh \\ Department of Neurosurgery, Samsung Medical Center, Sungkyunkwan University School of Medicine, Seoul, Korea
}

\begin{abstract}
Superficial siderosis of the central nervous system (SSCNS) is a rare disease characterized by hemosiderin deposition on the surface of the central nervous system. We report a case of SSCNS originating from the thoracic spine, presenting with neurological deficits including, sensorineuronal hearing loss, ataxia, and corticospinal and dorsal column tract signs. The patient underwent dural repair with an artificial dural patch. Clinical findings were elicited by neurological examination, imaging studies, and intraoperative findings, and these were addressed through literature review.
\end{abstract}

Key Words: Siderosis $\cdot$ Hemosiderin $\cdot$ Ataxia

\section{INTRODUCTION}

Superficial siderosis of the central nervous system (SSCNS) is an uncommon and unrecognized disorder caused by hemosiderin deposition in the subarachnoid space. This may lead to progressive neurological deficits, such as sensorineuronal hearing loss, cerebellar ataxia, signs of corticospinal tract dysfunction $^{7,15-17)}$.

Since the first pathologic clinical report of SSCNS was a case presented by Hamill in 1908, there have been approximately 300 reported cases of SSCNS in the literature ${ }^{3)}$. To date, only 21 cases of SSCNS originating from the spinal cord have been reported $^{2,6,19)}$.

Hemosiderin deposition in the subarachnoid space results from various pathologies, including tumors, meningocele and pseudomeningocele, vascular malformations, trauma, subarachnoid hemorrhage, spinal surgery, and brachial plexus, or nerve root injury. Spontaneous intracranial hypotension has also been associated with superficial siderosis ${ }^{20}$.

Here, we report a case of SSCNS originating from the thoracic spine with a dural defect. The patient presented with progressive gait disturbance after falling off a ladder. We also discuss the clinical symptoms, laboratory tests, imaging findings, and the association of SSCNS and dural defects.

- Received: February 7, 2016 • Revised: April 5, 2016

- Accepted: April 7, 2016

Corresponding Author: Eun-Sang Kim

Department of Neurosurgery, Samsung Medical Center, Sungkyunkwan University School of Medicine, Seoul 06351, Korea

Tel: +82-2-3410-3499, Fax: +82-2-3410-0048

E-mail: est.kim@samsung.com

$\otimes$ This is an open access article distributed under the terms of the Creative Commons Attribution Non-Commercial License (http://creativecommons.org/licenses/by-nc/4.0/) which permits unrestricted non-commercial use, distribution, and reproduction in any medium, provided the original work is properly cited.

\section{CASE REPORT}

A 55-year-old male developed progressive gait disturbance after falling off a ladder in March 2011. He felt "off balance" and noticed over the next two years an increasing difficulty walking. He had also developed difficulty voiding and tinnitus in both ears. He had visited several hospitals and undergone studies with computed tomographic angiography, transfemoral cerebral angiography, and magnetic resonance imaging (MRI) examinations. At that time, abnormal findings were not detected.

In April 2014, he was referred to Samsung Medical Center for ongoing neurological abnormalities. The patient's neurological exam was notable for dysmetria on heel-to-shin testing, which was markedly worse with the eyes closed. Tandem gait was noted to be unsteady, with a positive Romberg. Pinprick and temperature sensation were reduced in the distal lower extremities. Strength was $5 / 5$ bilaterally in both lower extremities. Urodynamic studies revealed severe underactivity of the detrusor. Examination of the cerebrospinal fluid found the red blood cell count elevated at $15,250 / \mu \mathrm{L}$ along with a single siderophage.

The patient underwent lumbar spine MRI at Samsung Medical Center, when the T2-weighted image (T2WI) demonstrated characteristic hypointensity outlining the spinal cord (Fig. 1). Additionally, adhesive arachnoiditis was found at the cauda equina, likely a result of previous subarachnoid hemorrhage. On brain MRI, hemosiderin deposition was seen in the sylvian fissures, posterior fossa and basal cistern (Fig. 2). CT myleography was performed to demonstrate the site of dural defect. After the skin area has been numbed, the dye is injected into the spinal sac, then the table is slowly rotated in a circular motion, first down at the head end for approximately 4 to 6 minutes, then rotated up at the head end for the same duration. The study showed a dural defect at T5, through which 


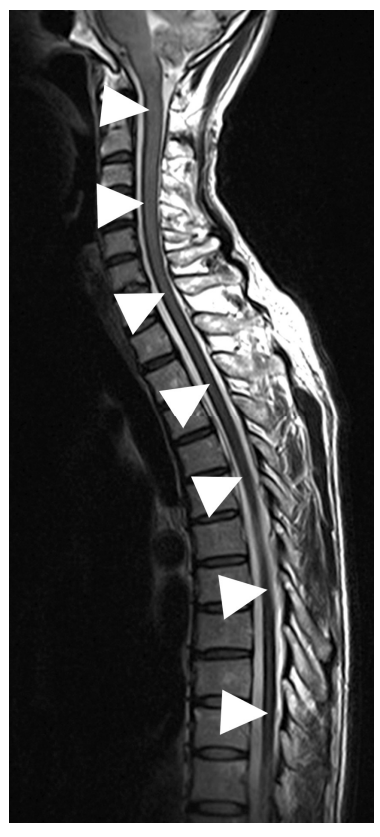

Fig. 1. Magnetic resonance scan of the cervical spine. Marginal hypointensity along the spinal cord was seen in the T2-weighted image (arrowheads).

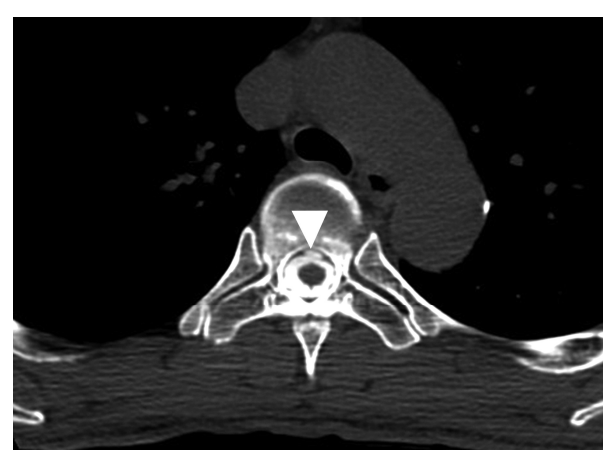

Fig. 2. Magnetic resonance scan of brain. Findings of hemosiderin deposition were seen around the crest of the cerebellar folia (arrowhead).

transdural accumulation of contrast media at the T3-T5 level was observed (Fig. 3).

Under monitoring of somatosensory and motor evoked potential (MEP), the patient underwent surgery to repair the dural defect. Hemilaminectomy was performed at the left partial inferior T4 and partial superior T5. The spinal cord was carefully retracted using a Penfield No. 4 dissector (Symmetry Surgical, Antioch, TN, USA). A dural hole was visualized at the ventral portion of T5 (Fig. 4A) and fragile vessels were found surrounding the surface of the dural defect. We resected a tiny piece of arachnoid at the dural defect for histological examination, after which primary closure of the dura was attempted with 6-0 polypropylene (Ehticon, Somerville, NJ, USA), using a single stitch. Additionally, a $0.5 \times 0.5-\mathrm{mm}$ patch of artificial dura (Aesculap Inc., Central Valley, PA, USA) was anchored to the remaining dural defect (Fig. 4B). At this time, we noticed the loss of the MEP at the left lower extremity and paused the

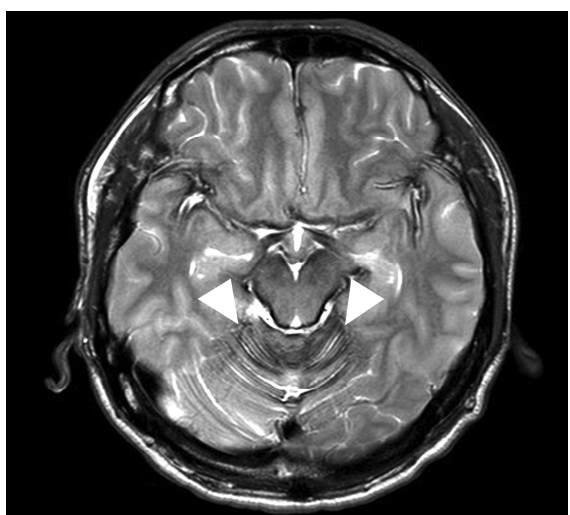

Fig. 3. Computed tomography (CT) myelogram of the thoracic level. Preoperative axial CT myelogram showing fluid collection in the spinal canal (arrowheads).
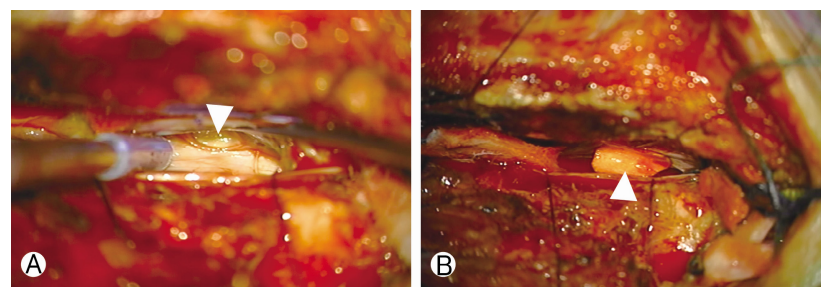

Fig. 4. Operative microscopic view. (A) The dural hole at the ventral portion of the spinal cord (arrowhead), (B) A dural patch was anchored to the remaining dural defect (arrowhead).

procedure for 20 minutes, before resuming closure of the dura using 6-0 polypropylene (Ehticon). We did not recover the MEP during the remainder of the procedure. Immediately after recovery from anesthesia, strength in the left lower-extremity was $0 / 5$. By postoperative day one, the strength had improved to $1 / 5$. After that, the patient showed graual improvement of the weakness. One month after the surgery, strength was $4+/ 4+$ bilaterally in both lower extremities.

Histological examination demonstrated chronic inflammation of the arachnoid membrane. Follow-up MRI at 2 months after surgery showed no significant interval change in superficial siderosis along the spinal cord. Six months after surgery, gait disturbance and voiding difficulty had progressed, though tinnitus and diplopia were slightly improved.

\section{DISCUSSION}

SSCNS is a rare condition characterized by the deposition of hemosiderin in the subpial layers of the central nervous system (CNS), as a result of chronic subarachnoid bleeding. The first pathologic clinical presentation of SSCNS was a case reported by Hamill in 1908, but only 40 cases appeared in the literature before the widespread use of MRI examination. After that, an additional 230 cases have been reported, all showing 
a strikingly similar T2WI hypointensity on MRI examination ${ }^{3)}$. To date, only 21 cases of SSCNS originating from the spinal cord have been reported. Thirteen of these involved a spinal dural defect, while the other eight involved meningoceles ${ }^{2,6}$. Males are affected more frequently than females with a ratio of $2: 1^{15)}$. One case series report, which included a review of the literature, reported that the mean age of patients with SSCNS was 59 years $^{10)}$.

Idiopathic bleeding is the main cause of subarachnoid bleeding, accounting for 35\% of all cases; this is followed by CNS tumors (15\%), head trauma (13\%), and arteriovenous malformation $(9 \%)^{7}$. Spontaneous intracranial hypotension has also been associated with superficial siderosis ${ }^{20}$.

The pathophysiology of SSCNS primarily results from hemosiderin desposition in the subaracnoid space. Hemosiderin is found within cells and appears to be a complex of ferritin, denatured ferritin and other materials. Ferritin is known to be involved in the CNS tissue response to CSF hemoglobin and hemoglobin breakdown products ${ }^{13)}$. Animal studies suggest that the ferritin repressor protein known as immunoreactive Bergmann glia and ferritin-containing microglia are involved in the conversion of heme to ferritin to hemosiderin. Recurrent subarachnoid hemorrhage leads to an overproduction of hemoglobin degradation products, including free iron $^{8)}$. The free iron from hemosiderin catalyzes the oxidation of hydrogen peroxide to superoxide, causing lipid peroxidation, membrane dysfunction, and ultimately cell death. Microscopically, the end result is neuronal loss, reactive gliosis, and axonal demyelination". Because of the microanatomy of the CNS and the specific cerebrospinal fluid flow pattern, hemosiderin deposition generally occurs in the superior vermis, crest of the cerebellar folia, basal frontal lobe, temporal cortex, brainstem, spinal cord, nerve roots, and cranial nerves I and VIII ${ }^{7,915)}$. In the present case, hemosiderin deposition was limited to the spinal cord and infratentorial area. Fragile vessels were found surrounding the surface of the dural defect, and were assumed to be the cause of chronic repetitive bleeding that eventually resulted in SSCNS. Even in the first series of SSCNS cases described by Bracchi et al. ${ }^{3)}$, many posttraumatic events were noted. The fragile vessels developing on the unrepaired dura were implicated as the probable source of chronic microhemorrhages ${ }^{3)}$.

The neurologic deficits caused by hemosiderin deposition are irreversible. The latent period of SSCNS ranges from 4 months to 30 years $^{7}$. Common clinical findings include sensorineuronal hearing loss (95\%), progressive cerebellar ataxia $(88 \%)$, and pyramidal tract signs $(76 \%)^{15-17)}$. In the present case, the patient exhibited ataxia, diplopia, voiding difficulty and bilateral tinnitus with preserved hearing. Though the current patient did not experience hearing deficits, long-term followup is required, as the development of sensorineuronal deafness is common.

Prior to the widespread use of MRI, the diagnosis of SSCNS depended on postmortem examination or surgical biopsy ${ }^{7}$. Currently, MRI is the diagnostic modality of choice. T2WI
MRI reveals a pathognomonic linear hypointensity due to hemosiderin deposition around the brainstem, cerebellum, spinal cord, and cauda eaquina". Recently, an association between dural defects in the spine and SSCNS has been suggested. Sometimes, a longitudinally fluid-filled collection in the spinal canal is observed with MRI. Kumar et al. ${ }^{11)}$ have reported the efficacy of CT myelography in detecting the communication between the subarachnoid space and fluid collection. In the present case, CT myleography was performed to detect the site of dural defect and showed a dural defect at T5 with accumulation of contrast media (Fig. 2). Even though myelography is generally regarded as a safe procedure, close attention is necessary in the possibility of fatal complications such as seizure and rhabdomyolysis ${ }^{4}$.

Currently, there is no effective treatment for SSCNS. Medical management in the form of corticosteroids has been reportedly successful in a unique case associated with anti-Ri antibodies ${ }^{1)}$. Penicilamine and large doses of vitamin $\mathrm{C}$ and $\mathrm{E}$ have also been effective in certain patients ${ }^{14}$. Treatment with deferiprone, a lipid soluble iron chelator, has reduced hemosiderin deposition, as evidenced on MRI, in a cohort of 10 patients with SSCNS. However, prospectively designed efficacy studies are necessary to determine the clinical efficacy of deferiprone in $\mathrm{SSCNS}^{15)}$.

As there is no definitive treatment for SSCNS, surgical closure of the defect remains the most reliable method, effectively halting ongoing bleeding from dural defects associated with SSCNS $^{6}$. However, surgery has been effective in very few patients, with many patients experiencing aggravated neurological deficiencies following surgery. In the present case, the aggravation of gait disturbance and voiding difficulty is likely to have been caused by ongoing SSCNS. Posti et al. ${ }^{19)}$ performed a PubMed search using the keywords "superficial siderosis" associated with the keywords "surgery", "surgical," "operative", and "treatment". Of the 29 surgically managed cases of SSCNS, clinical improvement was seen in only 5 cases, with another 5 cases endorsing progressive symptoms following surgery. The remaining 19 cases endorsed neurological stability. Payer et al. ${ }^{18)}$ recently reported that the disease can progress secondarily without rebleeding after surgical closure. Direct suture can be difficult to perform when the defect is located in the ventral portion, as in the present case. We performed additional anchoring sutures with an artificial dura, in which cases, fibrin glue, collagen sponges, and muscle or fat grafting can also be used in repair ${ }^{2,5,11,12)}$.

\section{CONCLUSION}

Surgical repair of the dural defect is effective to halt cerebrospinal fluid leakage and subarachnoid hemorrhaging. If there are long histories of SSCNS, the symptoms can deteriorate even after a complete dural closure. Therefore, early diagnosis, precise detection of the dural defect location, and prompt surgical repair are important for minimizing the neurologic impairment. 


\section{CONFLCT OF INTEREST}

No potential conflict of interest relevant to this article was reported.

\section{REFERENCES}

1. Angstwurm K, Schielke E, Zimmer C, Kivelitz D, Weber JR: Superficial siderosis of the central nervous system: response to steroid therapy. J Neurol 249:1223-1225, 2002

2. Boncoraglio GB, Ballabio E, Erbetta A, Prada F, Savoiardo M, Parati EA: Superficial siderosis due to dural defect with thoracic spinal cord herniation. J Neurol Sci 312:170-172, 2012

3. Bracchi M, Savoiardo M, Triulzi F, Daniele D, Grisoli M, Bradac GB, et al: Superficial siderosis of the CNS: MR diagnosis and clinical findings. AJNR Am J Neuroradiol 14:227-236, 1993

4. Cha KC, Kim JH, Moon BG, Kang HI, Lee SJ: Myelography Induced Fatal Complications; Seizure and Rhabdomyolysis. Korean J Spine 7:192-194, 2010

5. Cheng CY, Chen MH, Wang SJ, Lin KP: A proposed mechanism of superficial siderosis supported by surgical and neuroimaging findings. Med Hypotheses 76:823-826, 2011

6. Egawa S, Yoshii T, Sakaki K, Inose H, Kato T, Kawabata S, et al: Dural closure for the treatment of superficial siderosis. J Neurosurg Spine 18:388-393, 2013

7. Fearnley JM, Stevens JM, Rudge P: Superficial siderosis of the central nervous system. Brain 118(Pt 4):1051-1066, 1995

8. Koeppen AH, Dickson AC, Chu RC, Thach RE: The pathogenesis of superficial siderosis of the central nervous system. Ann Neurol 34:646-653, 1993

9. Kumar N: Neuroimaging in superficial siderosis: an in-depth look. AJNR Am J Neuroradiol 31:5-14, 2010
10. Kumar N, Cohen-Gadol AA, Wright RA, Miller GM, Piepgras DG, Ahlskog JE: Superficial siderosis. Neurology 66:1144-1152, 2006

11. Kumar N, Lane JI, Piepgras DG: Superficial siderosis: sealing the defect. Neurology 72:671-673, 2009

12. Kumar N, Lindell EP, Wilden JA, Davis DH: Role of dynamic CT myelography in identifying the etiology of superficial siderosis. Neurology 65:486-488, 2005

13. Leibold EA, Guo B: Iron-dependent regulation of ferritin and transferrin receptor expression by the iron-responsive element binding protein. Annu Rev Nutr 12:345-368, 1992

14. Leussink VI, Flachenecker P, Brechtelsbauer D, Bendszus M, Sliwka U, Gold R, et al: Superficial siderosis of the central nervous system: pathogenetic heterogeneity and therapeutic approaches. Acta Neurol Scand 107:54-61, 2003

15. Levy M, Turtzo C, Llinas RH: Superficial siderosis: a case report and review of the literature. Nat Clin Pract Neurol 3:54-58, 2007

16. Matsumoto S, Kang Y, Sato S, Kawakami Y, Oda Y, Araki M, et al: Spinal meningeal melanocytoma presenting with superficial siderosis of the central nervous system. Case report and review of the literature. J Neurosurg 88:890-894, 1998

17. Miliaras G, Bostantjopoulou S, Argyropoulou M, Kyritsis A, Polyzoidis K: Superficial siderosis of the CNS: report of three cases and review of the literature. Clin Neurol Neurosurg 108: 499-502, 2006

18. Payer M, Sottas C, Bonvin C: Superficial siderosis of the central nervous system: secondary progression despite successful surgical treatment, mimicking amyotrophic lateral sclerosis. Case report and review. Acta Neurochir (Wien) 152:1411-1416, 2010

19. Posti JP, Juvela S, Parkkola R, Roine S: Three cases of superficial siderosis of the central nervous system and review of the literature. Acta Neurochir (Wien) 153:2067-2073, 2011

20. Schievink WI, Wasserstein P, Maya MM: Intraspinal hemorrhage in spontaneous intracranial hypotension: link to superficial siderosis? Report of 2 cases. J Neurosurg Spine 24:454-456, 2016 